Yadav et al.

Supporting Information (SI) for

\title{
Kinetically Blocked Stable 5,6:12,13-Dibenzozethrene: A Laterally $\pi$-Extended Zethrene with Enhanced Diradical Character
}

Priya Yadav,${ }^{\dagger}$ Soumyajit Das,${ }^{\dagger}$ Hoa Phan,${ }^{\dagger}$ Tun Seng Herng,${ }^{\dagger}$ Jun Ding,${ }^{\ddagger}$ Jishan $\mathrm{Wu}^{*}, \dagger, \S$

${ }^{\dagger}$ Department of Chemistry, National University of Singapore, 3 Science Drive 3, Singapore, 117543

Department of Materials Science \& Engineering, National University of Singapore, 119260, Singapore

${ }^{\S}$ Institute of Materials Research and Engineering, A*STAR, 2 Fusionopolis Way, Innovis, \#08-03, Singapore, 117602

\section{Table of Contents}

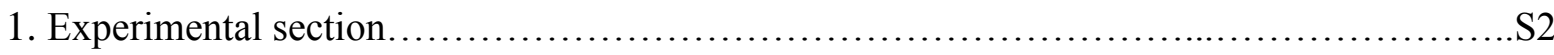

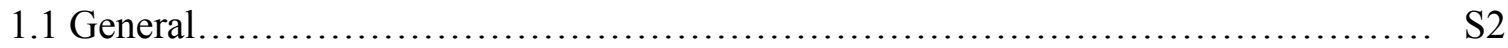

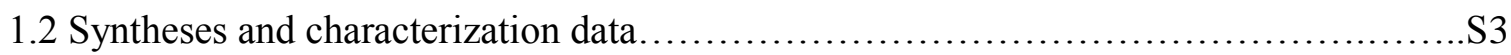

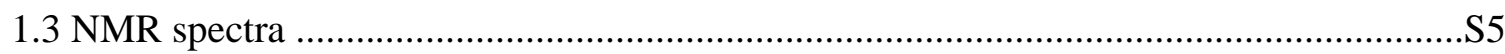

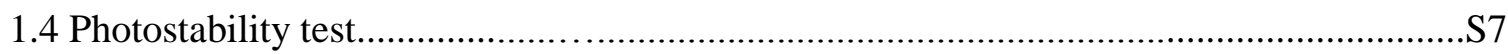

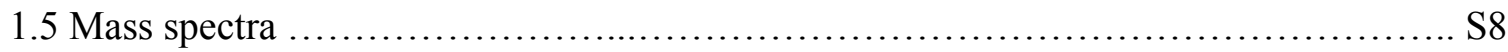

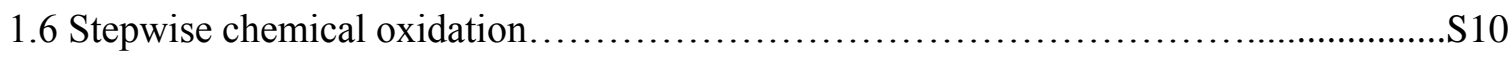

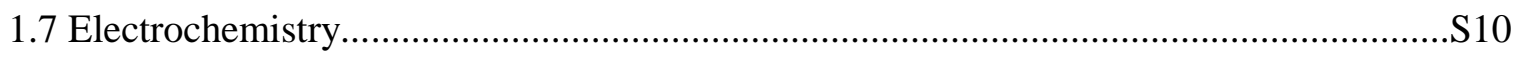

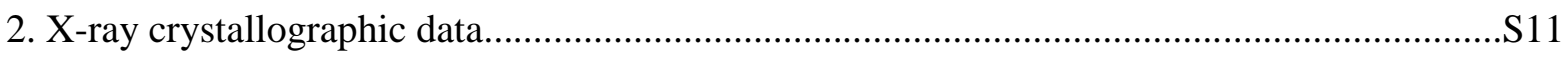

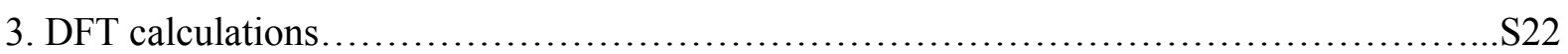


Yadav et al.

\section{Experimental Section}

\subsection{General}

All common reagents, and catalysts were purchased from commercial suppliers and used as received without further purification. Specific chemicals such as 2ethoxycarbonylphenylboronic acid, methanesulfonic acid and $n$-butyllithium were purchased from Sigma-Aldrich; 6,12-dibromochrysene was purchased from Luminescence Technology Corp (Taiwan); triisopropylsilyl(TIPS)acetylene was purchased from Alfa-aesar. Tetrahydrofuran was distilled over sodium/benzophenone prior to use. The ${ }^{1} \mathrm{H}$ NMR and ${ }^{13} \mathrm{C}$ NMR spectra were recorded in $\mathrm{CDCl}_{3}$ (deacidified) and $\left[\mathrm{D}_{8}\right] \mathrm{THF}$ on either Bruker DPX 300 or DRX 500 NMR spectrometers with tetramethylsilane (TMS) as the internal standard. ESR spectra were obtained with a JEOL (FA200) spectrometer. High resolution APCI mass spectra were recorded on Bruker amazonX mass spectrometer. UV-vis-NIR absorption was recorded on Shimadzu UV-3600Plus. The electrochemical measurements on $\mathrm{CH}$ Instruments were carried out in dry DCM with $0.1 \mathrm{M}$ tetrabutylammonium hexafluorophosphate $\left(\mathrm{TBAPF}_{6}\right)$ as the supporting electrolyte at a scan rate of $100 \mathrm{mV} / \mathrm{s}$ at room temperature under the protection of nitrogen. A gold disk was used as working electrode, platinum wire was used as counter electrode, and $\mathrm{Ag} / \mathrm{AgCl}$ (3M KCl solution) was used as reference electrode. The potential was externally calibrated after each experiment, against the ferrocene/ferrocenium couple.

A Quantum Design 7 Tesla SQUID-VSM system was available for the magnetic measurements in this work. Powder sample with a weight of 5-10 mg was sealed in a plastic capsule. The magnetic susceptibility was measured in the temperature range of 2 to $380 \mathrm{~K}$ with an applied field of $0.5 \mathrm{~T}$. After correction of diamagnetic contributions from the sample, using tabulated constants, ${ }^{1}$ sample holder and paramagnetic contamination, the magnetic data were fitted with Bleaney-Bowers equation $(\chi=$ $\left.\frac{2 N \beta^{2} g^{2}}{3 k T}\left[1+\frac{1}{3} \exp \left(\frac{J_{S-t}}{k T}\right)\right]^{-1}\right)$. 
Yadav et al.

\subsection{Syntheses and characterisation data}

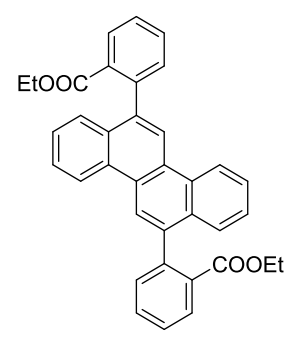

Compound 3. An oven-dried two-necked round bottom flask was charged with 1 (100 mg, $0.25 \mathrm{mmol})$, 2-ethoxycarbonylphenylboronic acid (2, $148 \mathrm{mg}, 0.625 \mathrm{mmol}), 2 \mathrm{M}$ aq. $\mathrm{K}_{2} \mathrm{CO}_{3}(1$ $\mathrm{ml})$ and freshly distilled tetrahydrofuran $(10 \mathrm{ml})$, then purged with argon for 30 mins. $\mathrm{Pd}\left(\mathrm{PPh}_{3}\right)_{4}(29 \mathrm{mg}, 10 \mathrm{~mol} \%)$ was added under argon. This resultant mixture was then heated at $85{ }^{\circ} \mathrm{C}$ for $24 \mathrm{~h}$. After cooling to room temperature, THF was removed on rotatory evaporator and water was added to it. After extraction of the reaction mixture with dichloromethane followed by drying over sodium sulphate, solvent was evaporated to dryness. The crude mixture obtained was purified by silica gel column chromatography (hexanes : ethyl acetate $=90: 10)$ to give the desired compound $\mathbf{3}$ as white solid $(80 \mathrm{mg}, 60 \%$ yield). ${ }^{1} \mathrm{H}$ NMR $\left(\mathrm{CDCl}_{3}, 300 \mathrm{MHz}\right): \delta \mathrm{ppm} 8.79(\mathrm{~d}, J=8.3 \mathrm{~Hz}, 2 \mathrm{H}), 8.58(\mathrm{~s}, 2 \mathrm{H}), 8.14(\mathrm{~d}, J=$ $7.6 \mathrm{~Hz}, 2 \mathrm{H}), 7.48-7.72(\mathrm{~m}, 12 \mathrm{H}), 3.80-3.68(\mathrm{~m}, 4 \mathrm{H}), 0.48(\mathrm{t}, J=7.1 \mathrm{~Hz}, 3 \mathrm{H}), 0.37$ (t, $J=7.1$ $\mathrm{Hz}, 3 \mathrm{H}) .{ }^{13} \mathrm{C} \mathrm{NMR}\left(\mathrm{CDCl}_{3}, 125 \mathrm{MHz}\right): \delta 167.8,167.6,141.6,141.4,139.1,139.0,132.2$, 132.2 , 131.9, 131.8, 131.7, 131.6, 130.2, 130.2, 127.8, 127.8, 127.1, 126.5, 126.5, 126.4, $126.4,126.3,126.3,123.3,123.3,121.1,121.0,60.6,60.5,29.7,13.1,12.9$. HRMS (APCI): calcd. for $\mathrm{C}_{36} \mathrm{H}_{28} \mathrm{O}_{4}[\mathrm{M}]^{+}, 524.1982$; found, 524.1975 (error $=+1.3$ )

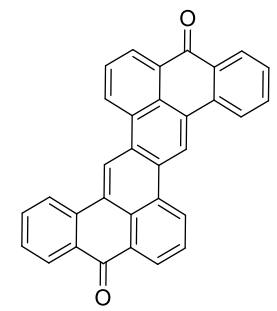

Compound 4. An oven-dried two-necked round bottom flask was charged with 3 (100 mg, $0.19 \mathrm{mmol})$ and methanesulphonic acid $(8 \mathrm{ml})$ under argon. This mixture was refluxed at 100 ${ }^{\circ} \mathrm{C}$ for $2 \mathrm{~h}$. After cooling to room temperature, $50 \mathrm{ml}$ water was added to it and stirred well for 1-2 h. The brown coloured precipitate was filtered under vacuum suction, and washed several times with water, and methanol and finally dried (70 mg, 85\% yield). Because of very poor solubility, ${ }^{1} \mathrm{H} \mathrm{NMR},{ }^{13} \mathrm{C}$ NMR and mass spectra could not be obtained and we directly proceeded to next step. 


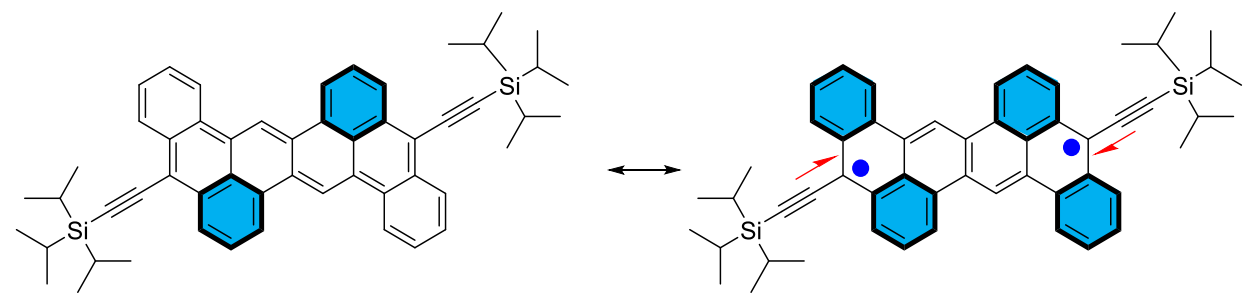

Compound 5. To a solution of triisopropylsilylacetylene $(252 \mathrm{mg}, 1.39 \mathrm{mmol})$ in dry tetrahydrofuran $(10 \mathrm{ml})$ at $0{ }^{\circ} \mathrm{C}, n$-BuLi $(1.6 \mathrm{M}$ in hexane, $0.9 \mathrm{ml}, 1.39 \mathrm{mmol})$ was added dropwise and stirred for 30 minutes. The diketone 4 (100 $\mathrm{mg}, 0.23 \mathrm{mmol})$ was subsequently added and the reaction mixture was warmed to room temperature and left for overnight. During this interval, $\mathbf{4}$ got completely soluble and solution became clear. Resulting solution was then diluted with $10 \mathrm{ml}$ of toluene and then anhydrous $\mathrm{SnCl}_{2}(263 \mathrm{mg}, 1.38 \mathrm{mmol}$ ) was added to it under argon atmosphere. After addition, colour of the reaction mixture became green instantaneously. This dark green coloured solution was stirred for additional $1 \mathrm{~h}$. Upon completion of reaction, monitored by TLC, solvent was removed and the residue was purified by silica gel column chromatography (hexanes : $\mathrm{DCM}=95: 5$ and finally increased to 85 : 15). Compound 5 was obtained as green solid (45 mg, 30\% yield). ${ }^{1} \mathrm{H}$ NMR ([D $]$ THF, 500 $\mathrm{MHz}): \delta 8.94(\mathrm{~s}, 2 \mathrm{H}), 8.64(\mathrm{~s}, 4 \mathrm{H}), 8.48(\mathrm{~s}, 4 \mathrm{H}), 7.68(\mathrm{~s}, 2 \mathrm{H}), 7.60-7.42(\mathrm{~m}, 4 \mathrm{H}), 1.34(\mathrm{~s}$, $42 \mathrm{H}) .{ }^{13} \mathrm{C} \mathrm{NMR}\left(\mathrm{CDCl}_{3}, 125 \mathrm{MHz}\right)$ : No signal for dibenzozenthrene backbone was found after scanning for overnight ( $16 \mathrm{mg}$ in $\left.0.6 \mathrm{~mL} \mathrm{THF-} d_{8}\right)$ due to the significant diradical nature of compound 5. Further proof of the diradical nature was derived from strong and featureless ESR signal $\left(g_{\mathrm{e}}=2.0028\right)$ at room temperature that clearly supports the extensive delocalization of spin on the entire dibenzozethrene core. HRMS (APCI): calcd. for $\mathrm{C}_{54} \mathrm{H}_{59} \mathrm{Si}_{2}$ $[\mathrm{M}+\mathrm{H}]^{+}, 763.4150$; found, 763.4152 (error $=-0.3$ ). 


\subsection{NMR Spectra}

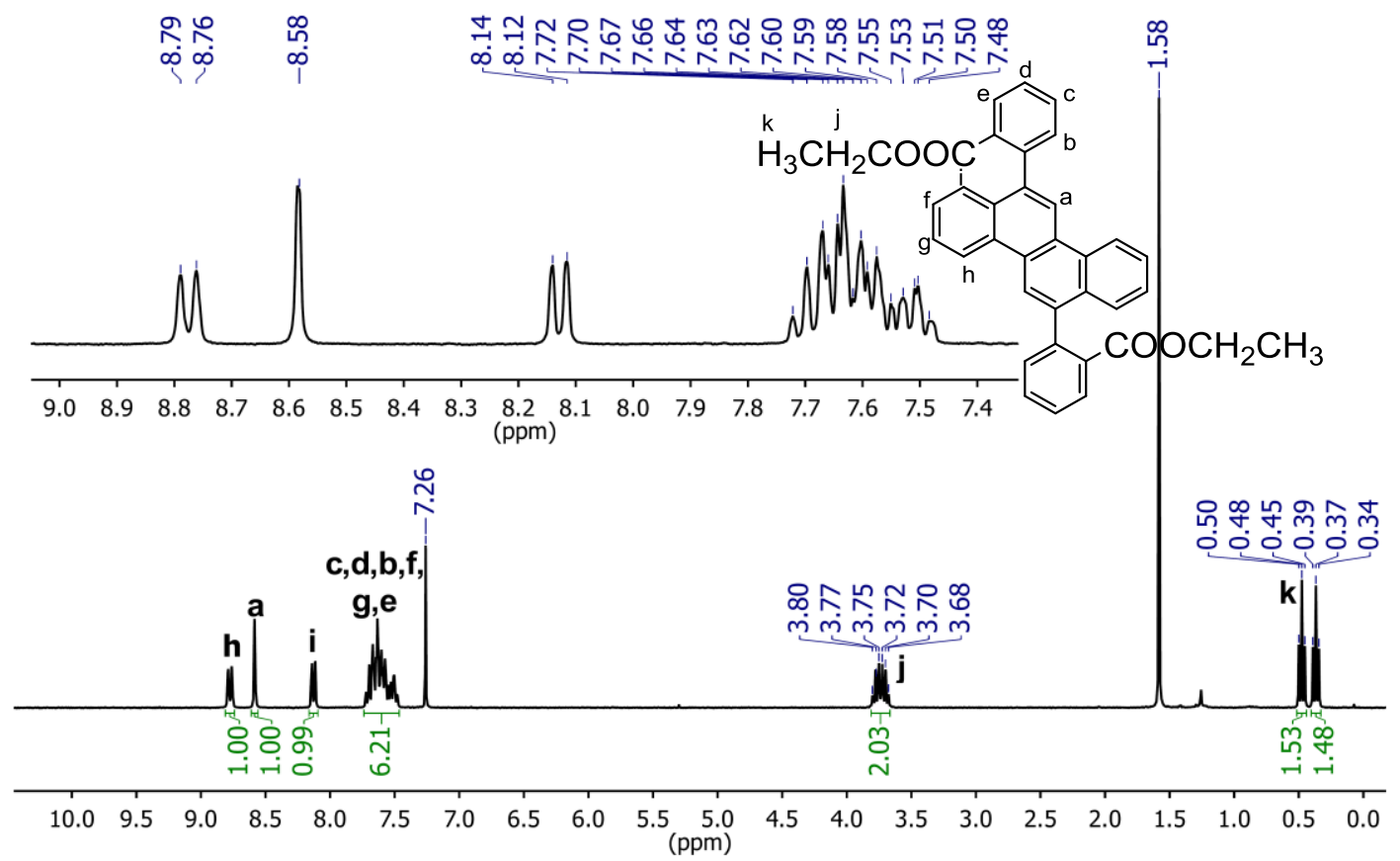

Figure S1. ${ }^{1} \mathrm{H}$ NMR spectra of compound $3\left(300 \mathrm{MHz}, \mathrm{rt}, \mathrm{CDCl}_{3}\right)$.

œ 눈

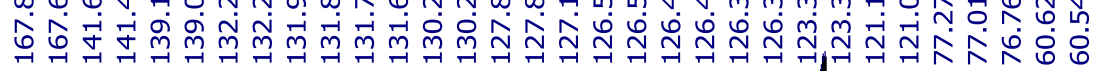

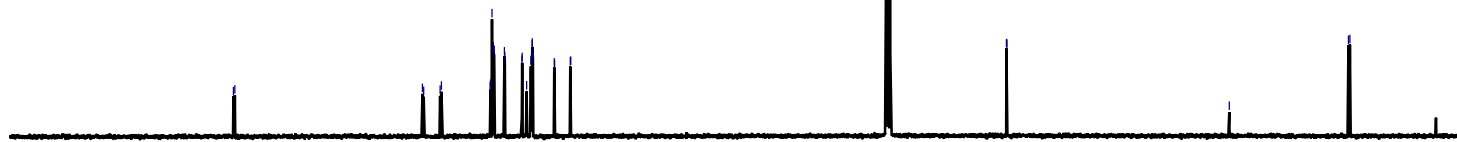

$\begin{array}{llllllllllllllllllll}190 & 180 & 170 & 160 & 150 & 140 & 130 & 120 & 110 & \begin{array}{c}100 \\ (\mathrm{ppm})\end{array} & 90 & 80 & 70 & 60 & 50 & 40 & 30 & 20 & 10 & 0\end{array}$

Figure S2. ${ }^{13} \mathrm{C}$ NMR spectra of compound $3\left(125 \mathrm{MHz}, \mathrm{rt}, \mathrm{CDCl}_{3}\right)$. 


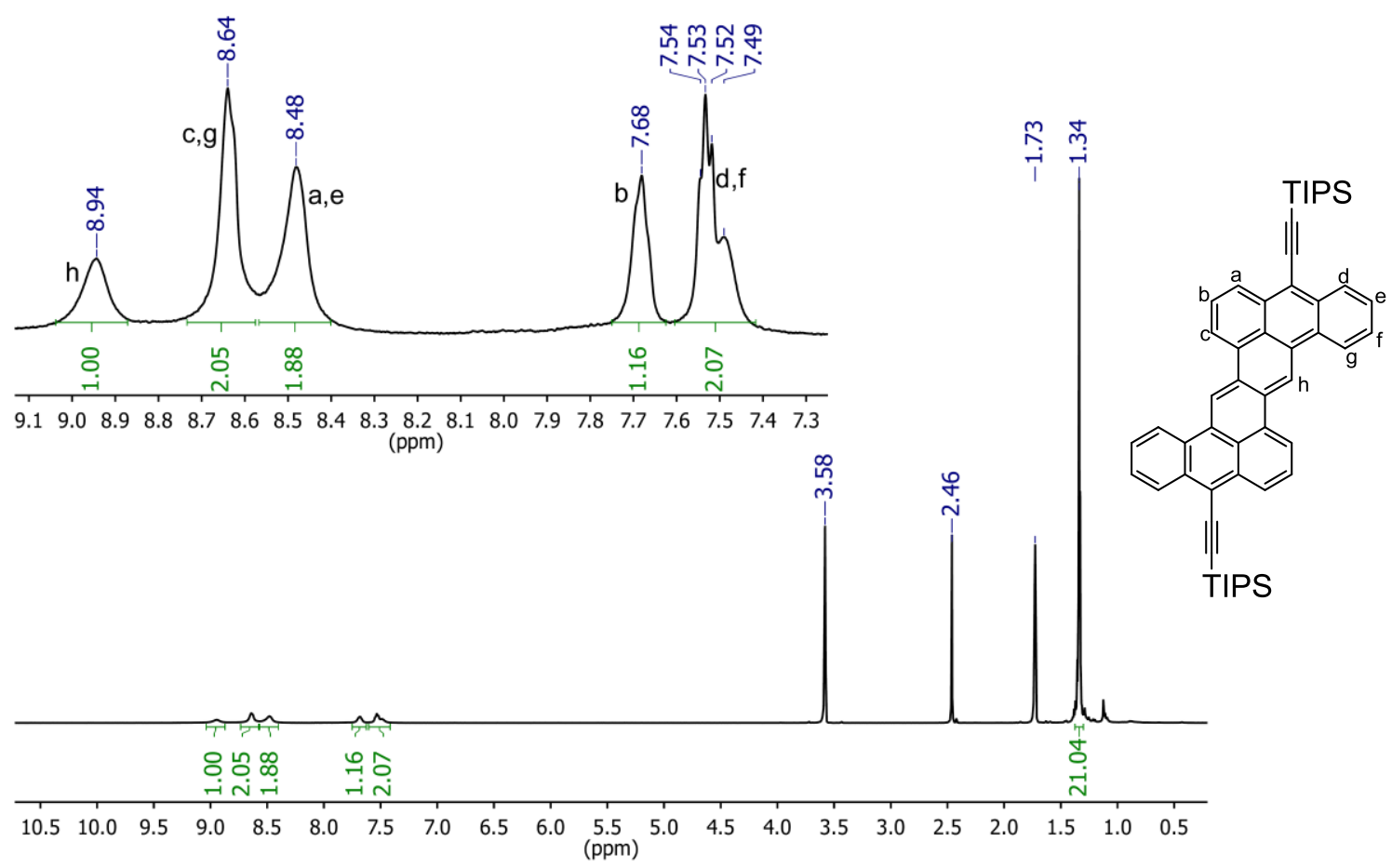

Figure S3. ${ }^{1} \mathrm{H}$ NMR spectra of compound $5\left(500 \mathrm{MHz}, \mathrm{rt},\left[\mathrm{D}_{8}\right] \mathrm{THF}\right)$.
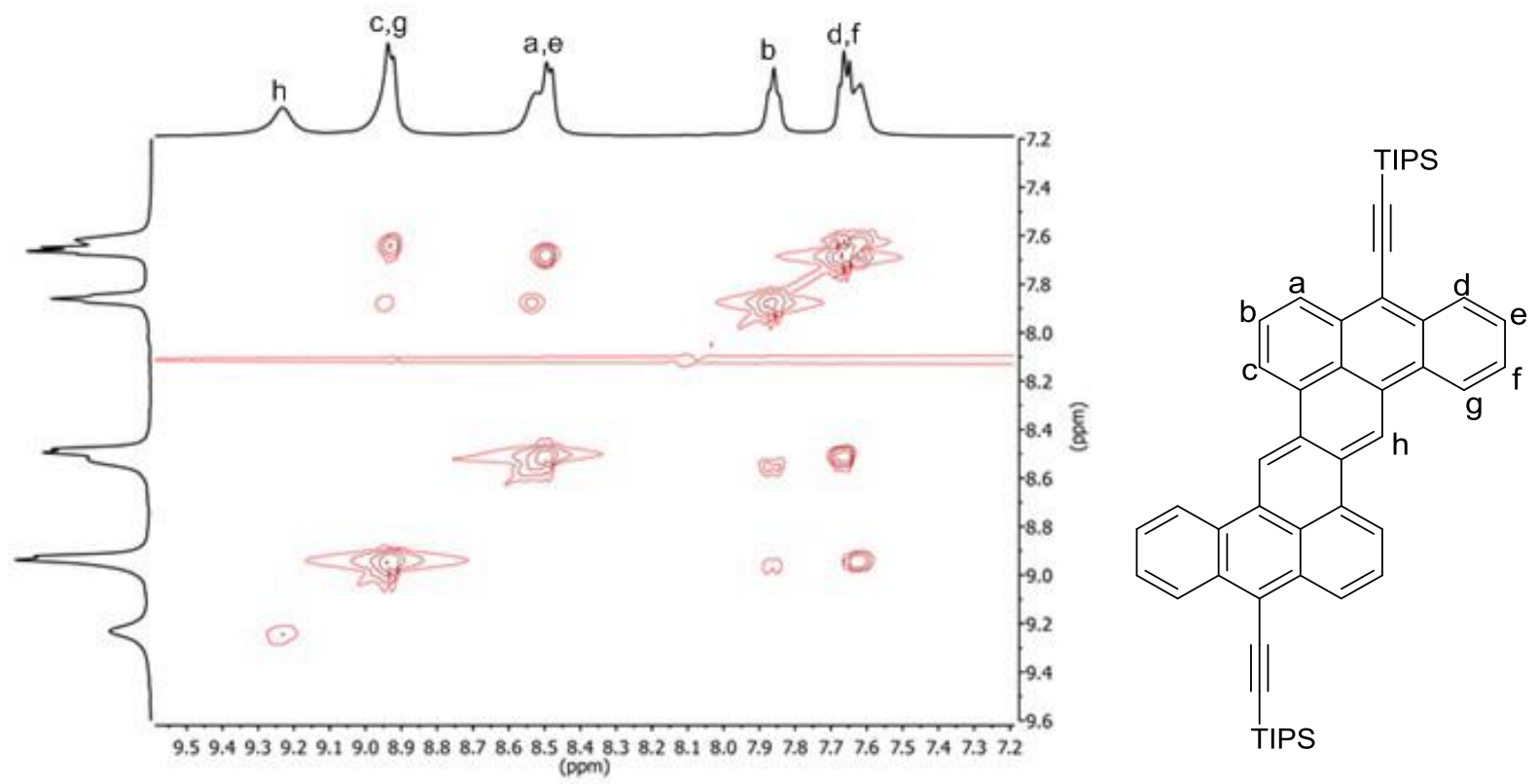

Figure S4. 2D COSY NMR of compound 5 with assignments (500 MHz, $\left.\left[\mathrm{D}_{8}\right] \mathrm{THF}\right)$. 
Yadav et al.

\subsection{Photostability test}

To check the photostability of final compound $\mathbf{5}$, solution of known concentration of $\mathbf{5}$ in dichloromethane was exposed in air. Irradiation of ambient light on it leads to gradual decomposition in solution, resulting in the decrease of the absorbance at longer wavelength (600-960 nm) region with simultaneous appearance of new absorbance bands in shorter wavelength region (300-500 $\mathrm{nm}$ ) (Figure S5a). By plotting absorbance of maximum wavelength $v s$ time, the half-life time $\left(t_{1 / 2}\right)$ of compound $\mathbf{5}$ was estimated as 33 hours (Figure S5b).
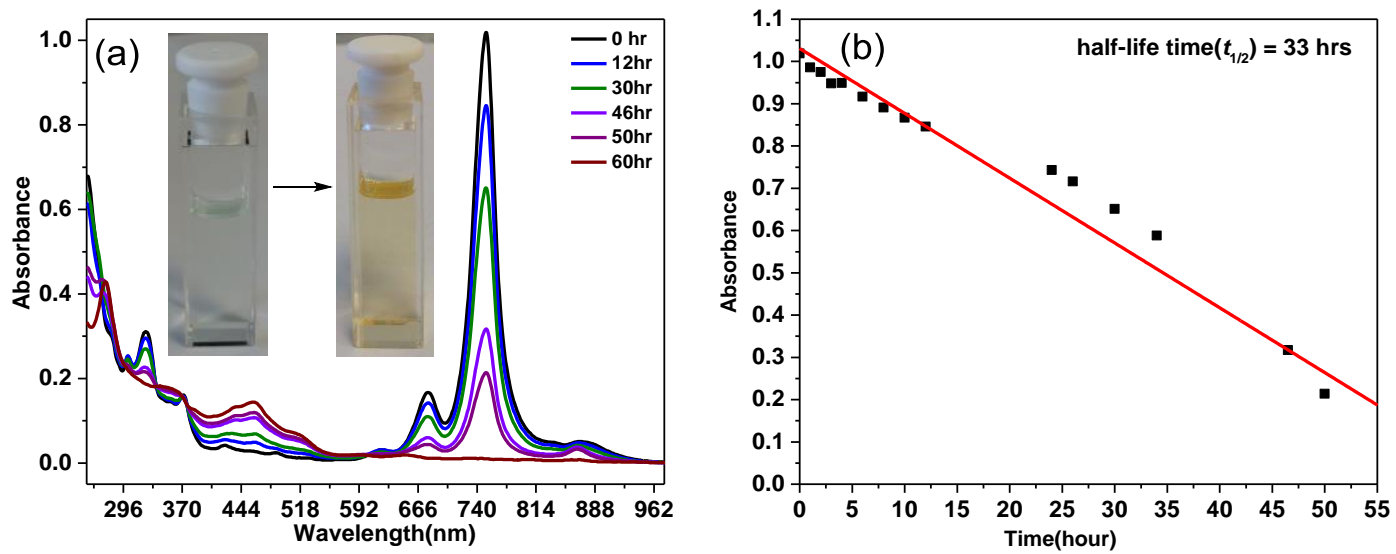

Figure S5. (a) Absorption spectral changes under ambient light for compound 5. (b) Absorption spectral changes at $751 \mathrm{~nm}$ with time under ambient light condition for compound 5. 
Yadav et al.

\subsection{Mass spectra}

\section{Mass Spectrum SmartFormula Report}

\begin{tabular}{|c|c|c|c|c|c|}
\hline \multicolumn{4}{|l|}{ Analysis Info } & Acquisition Date & 3/8/2016 3:12:46 PM \\
\hline $\begin{array}{l}\text { Analysis Name } \\
\text { Method } \\
\text { Sample Name } \\
\text { Comment }\end{array}$ & $\begin{array}{l}\text { D:IDatalChemistryl201 } \\
\text { YCH-150-1800.m } \\
\text { SM_1 } \\
\text { A/P Wu Jishan }\end{array}$ & Samplel201603\0308 & BSM_1-4.d & $\begin{array}{l}\text { Operator } \\
\text { Instrument / Ser\# }\end{array}$ & $\begin{array}{l}\text { default user } \\
\text { micrOTOF-Q II } 10269\end{array}$ \\
\hline \multicolumn{6}{|c|}{ Acquisition Parameter } \\
\hline $\begin{array}{l}\text { Source Type } \\
\text { Focus } \\
\text { Scan Begin } \\
\text { Scan End }\end{array}$ & $\begin{array}{l}\text { APCl } \\
\text { Not active } \\
50 \mathrm{~m} / \mathrm{z} \\
1800 \mathrm{~m} / \mathrm{z}\end{array}$ & $\begin{array}{l}\text { Ion Polarity } \\
\text { Set Capillary } \\
\text { Set End Plate Offset } \\
\text { Set Collision Cell RF }\end{array}$ & $\begin{array}{l}\text { Positive } \\
4500 \mathrm{~V} \\
-500 \mathrm{~V} \\
200.0 \mathrm{Vpp}\end{array}$ & $\begin{array}{l}\text { Set Nebulizer } \\
\text { Set Dry Heater } \\
\text { Set Dry Gas } \\
\text { Set Divert Valve }\end{array}$ & $\begin{array}{l}3.0 \mathrm{Bar} \\
200^{\circ} \mathrm{C} \\
6.0 \mathrm{l} / \mathrm{min} \\
\text { Waste }\end{array}$ \\
\hline
\end{tabular}

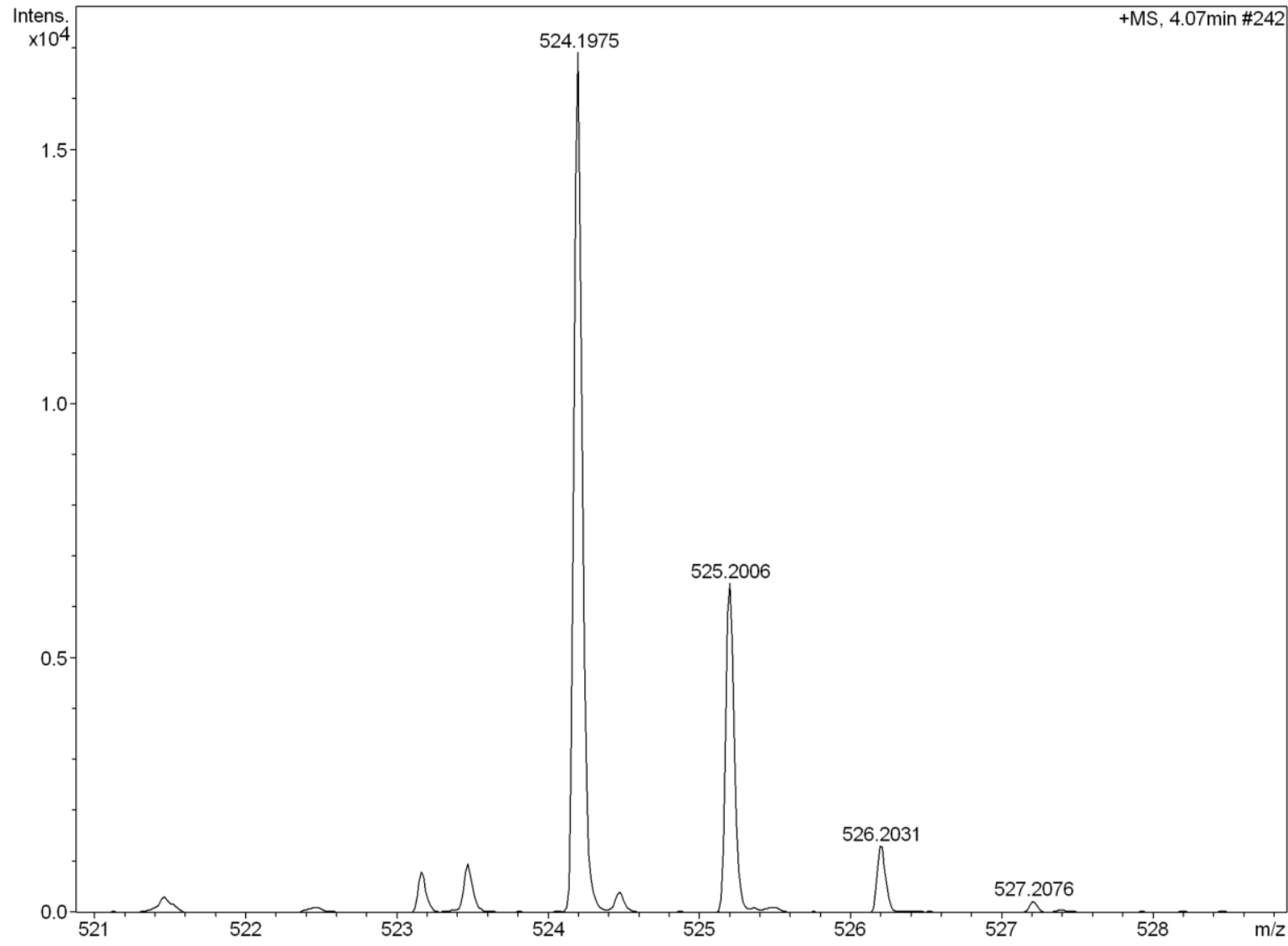

Figure S6. APCI-HRMS $[\mathrm{M}]^{+}$spectrum of compound 3 . 
Yadav et al.

\section{Mass Spectrum SmartFormula Report}

Analysis Info

Analysis Name D:IDatalChemistryl2015 Samplel20160110121-2IDBZ1TIPS-1.d

Method

YCH-150-1800.m

Acquisition Date 1/21/2016 2:25:39 PM

Sample Name DBZ1TIPS

Operator default user

Comment

A/P Wu Jishan

\section{Acquisition Parameter}

Source Type

Focus

$\mathrm{APCl}$

Scan Begin

Not active

$50 \mathrm{~m} / \mathrm{z}$

$1800 \mathrm{~m} / \mathrm{z}$

Instrument / Ser\# micrOTOF-Q II 10269

Scan End

Meas. $\mathrm{m} / \mathrm{z}$ \# Formula

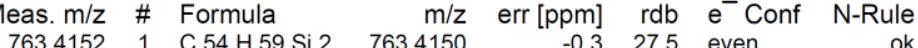

$\begin{array}{ll}\text { Ion Polarity } & \text { Positive } \\ \text { Set Capillary } & 4500 \mathrm{~V} \\ \text { Set End Plate Offset } & -500 \mathrm{~V} \\ \text { Set Collision Cell RF } & 200.0 \mathrm{Vpp}\end{array}$

Set Nebulizer

Set Dry Heater $\quad 200^{\circ} \mathrm{C}$

Set Dry Gas $\quad 6.01 / \mathrm{min}$

Set Divert Valve Waste

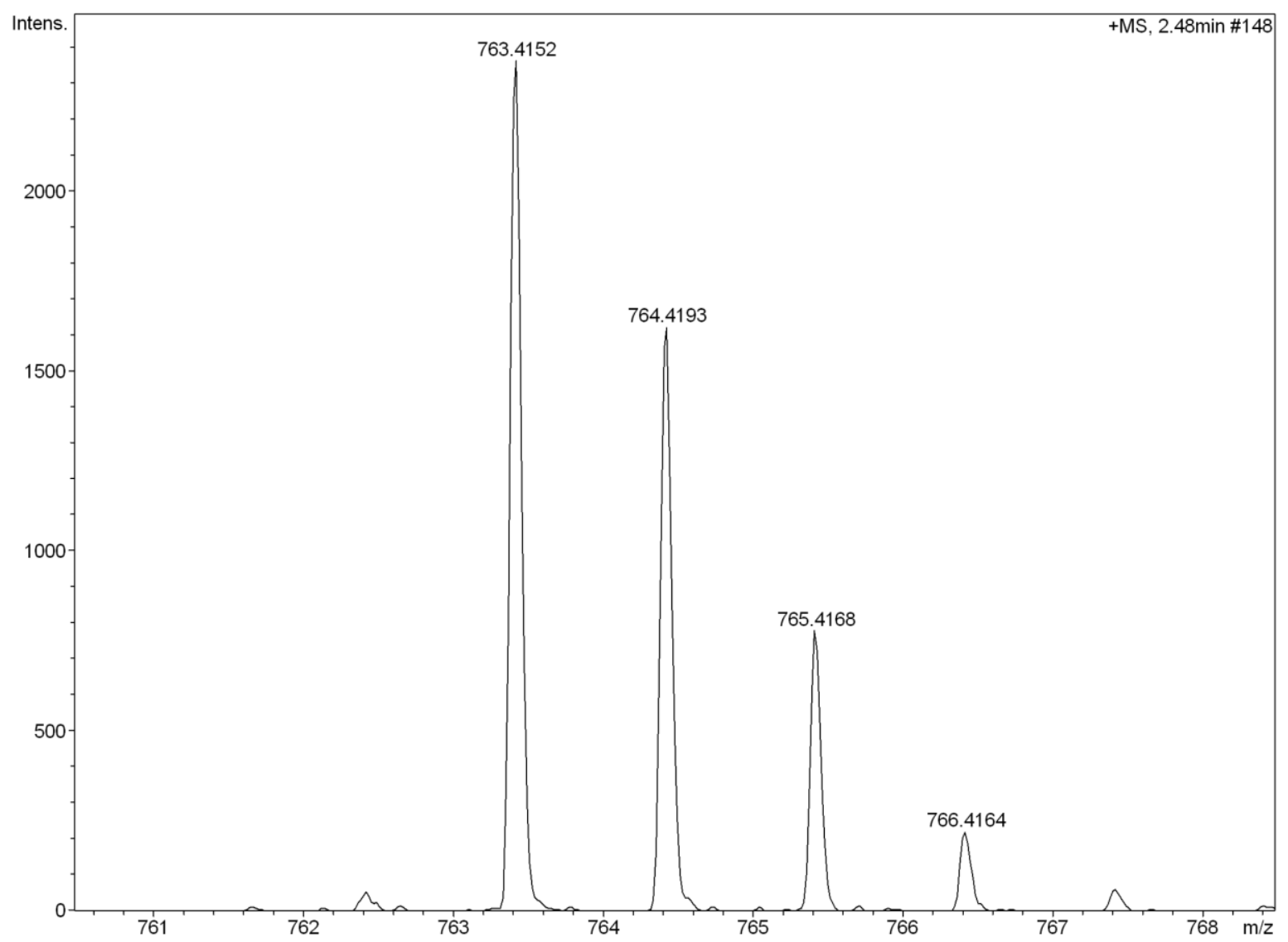

Figure S7. APCI-HRMS $[\mathrm{M}+\mathrm{H}]^{+}$spectrum of compound 5. 


\subsection{Stepwise chemical oxidation}
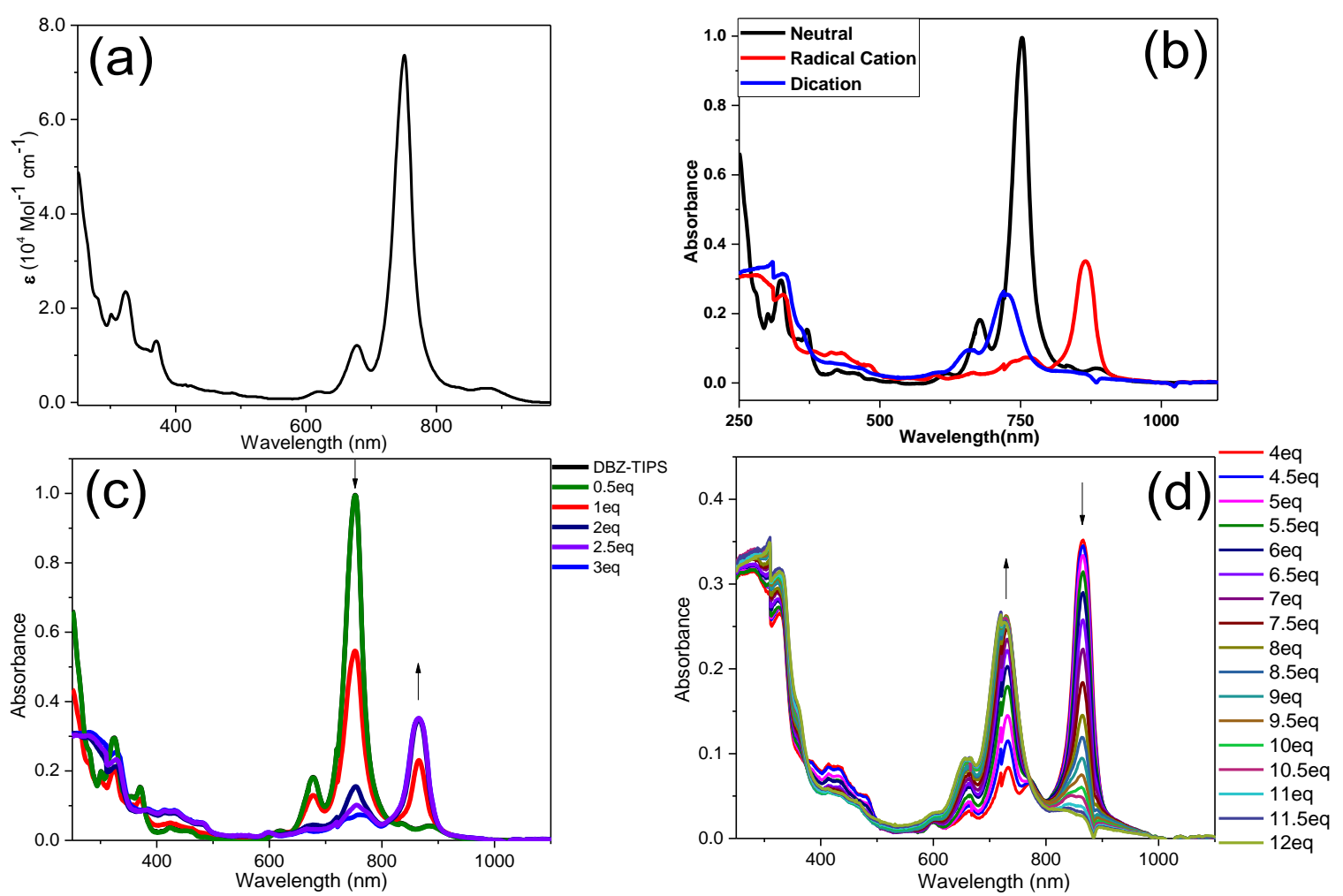

Figure S8. Titration with $\mathrm{SbCl}_{5}$ in dry DCM: (a) Absorption spectra of compound $\mathbf{5}$ in DCM.

(b) Absorption spectra for neutral, radical cation and dication state together of compound $\mathbf{5}$.

(c) Change in absorption spectra from neutral to radical cation for compound 5. (d) Change in absorption spectra from radical cation to dication.

\subsection{Electrochemistry}

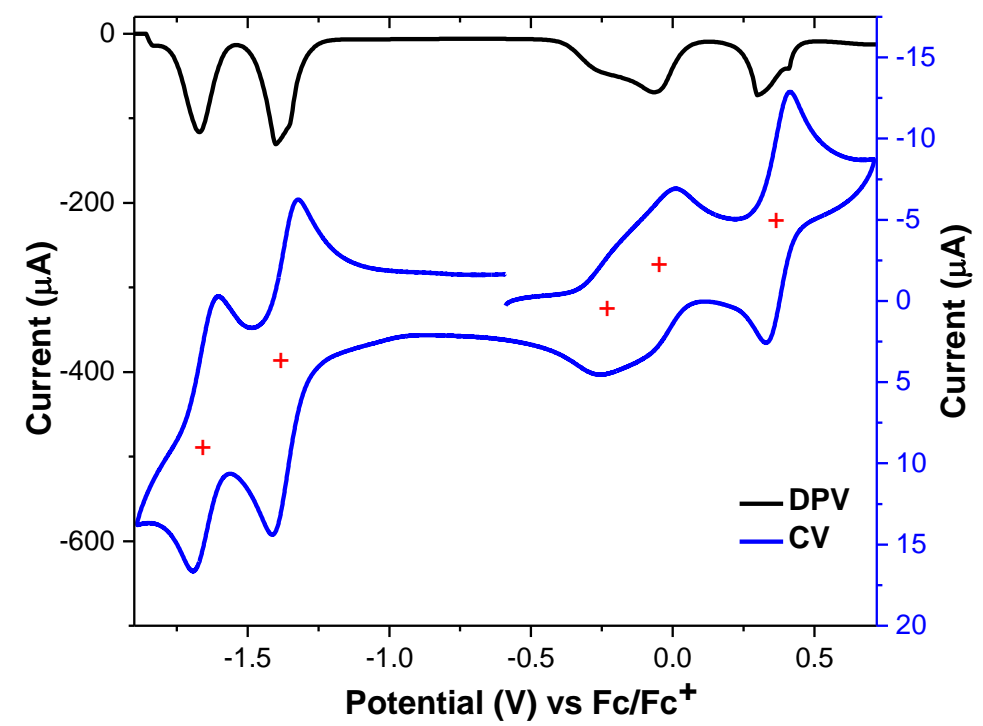

Figure S9. Cyclic voltammograms (CV) and differential pulse voltammograms (DPV) curves of 5. 


\section{X-ray Crystallographic Data}
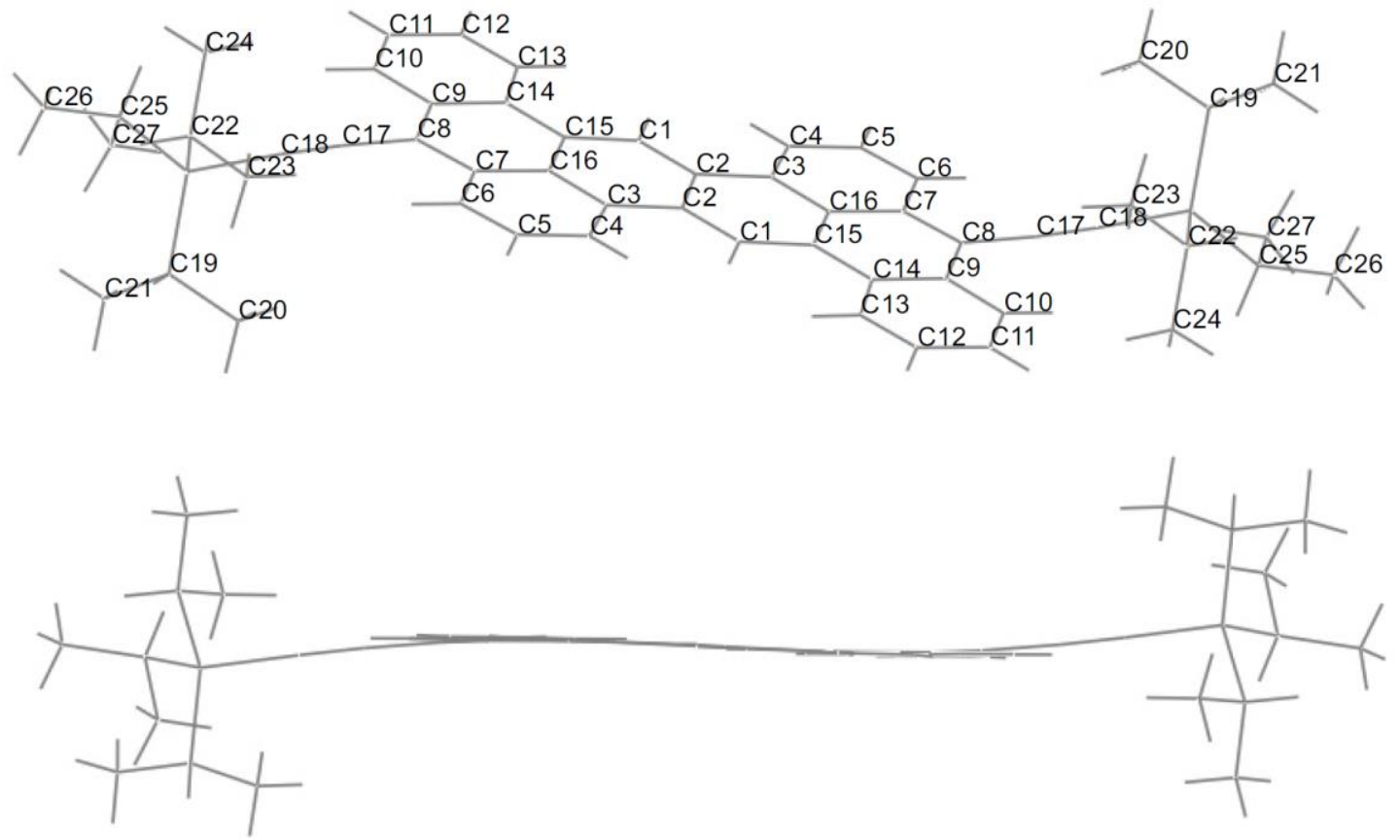

Figure S10. Crystallographic structure of 5, and its side view showing planar backbone.

Table S1. Crystal data and structure refinement for 5 .

CCDC Number

Chemical formula

Formula weight

Temperature

Wavelength

Crystal size

Crystal system

Space group

Unit cell dimensions

Volume

Z

Density (calculated)

Absorption coefficient
1475627

$\mathrm{C}_{31} \mathrm{H}_{37} \mathrm{OSi}$

$453.69 \mathrm{~g} / \mathrm{mol}$

100(2) K

$1.54178 \AA$

$0.145 \times 0.150 \times 0.167 \mathrm{~mm}$

Monoclinic

C $12 / \mathrm{c} 1$

$\mathrm{a}=36.1249(19) \AA \quad \alpha=90^{\circ}$

$\mathrm{b}=17.0168(9) \AA \quad \beta=93.726(3)^{\circ}$

$\mathrm{c}=8.3417(4) \AA \quad \gamma=90^{\circ}$
8

$1.178 \mathrm{~g} / \mathrm{cm}^{3}$

$0.951 \mathrm{~mm}^{-1}$ 
Yadav et al.

$\mathrm{F}(000) \quad 1960$

Theta range for data

collection

2.45 to $68.24^{\circ}$

Index ranges

$-38<=\mathrm{h}<=43,-20<=\mathrm{k}<=18,-9<=\mathrm{l}<=9$

Reflections collected $\quad 10778$

Independent reflections $\quad 4424[\mathrm{R}(\mathrm{int})=0.0523]$

Coverage of independent

reflections

$98 \%$

Absorption correction multi-scan

Max. and min. transmission 0.8740 and 0.8570

Refinement method Full-matrix least-squares on F2

Refinement program $\quad$ SHELXL-2014/7 (Sheldrick, 2014)

Function minimized $\quad \Sigma \mathrm{w}(\mathrm{Fo} 2-\mathrm{Fc} 2) 2$

Data / restraints /

parameters

4424 / 166 / 351

Goodness-of-fit on F2 1.072

Final $\mathrm{R}$ indices

$$
\begin{array}{ll}
3348 \text { data; } \mathrm{I}>2 \sigma(\mathrm{I}) & \mathrm{R} 1=0.0962, \mathrm{wR} 2= \\
& 0.2006 \\
& \mathrm{R} 1=0.1215, \mathrm{wR} 2= \\
\text { all data } & 0.2142
\end{array}
$$

Weighting scheme

$$
\mathrm{W}=1 /\left[\sigma^{2}\left(\mathrm{~F}_{\mathrm{o}}^{2}\right)+(0.0183 \mathrm{P})^{2}+50.0077 \mathrm{P}\right]
$$

where $\mathrm{P}=\left(\mathrm{F}_{\mathrm{o}}^{2}+2 \mathrm{~F}_{\mathrm{c}}^{2}\right) / 3$

Extinction coefficient $\quad 0.0000(0)$

Largest diff. peak and hole 0.511 and $-0.549 \mathrm{e}^{-3}$

R.M.S. deviation from mean $0.080 \mathrm{e}^{-3}$

Table S2. Atomic coordinates and equivalent isotropic atomic displacement parameters $\left(\AA^{2}\right)$ for $\mathbf{5}$, here $\mathrm{U}(\mathrm{eq})$ is defined as one third of the trace of the orthogonalized $\mathrm{U}_{\mathrm{ij}}$ tensor.

$\mathrm{x} / \mathrm{a}$

Si1 $\quad 0.35306(3)$

C1 0.49070(11)

C2 $0.48754(11)$ $\mathrm{y} / \mathrm{b}$

$0.82661(7)$

0.3963(3)

0.4764(3) $\mathrm{z} / \mathrm{c}$

0.56917(14)

0.0408(5)

$0.0428(5)$
$\mathrm{U}(\mathrm{eq})$

0.0210(3)

0.0192(9)

$0.0180(9)$ 


\begin{tabular}{|c|c|c|c|c|}
\hline & $\mathrm{x} / \mathrm{a}$ & $y / b$ & $\mathrm{z} / \mathrm{c}$ & $\mathrm{U}(\mathrm{eq})$ \\
\hline $\mathrm{C} 3$ & $0.45900(11)$ & $0.5163(3)$ & $0.1305(5)$ & $0.0169(9)$ \\
\hline $\mathrm{C} 4$ & $0.43331(11)$ & $0.4744(3)$ & $0.2149(5)$ & $0.0219(10)$ \\
\hline $\mathrm{C} 5$ & $0.40685(11)$ & $0.5127(3)$ & $0.3010(5)$ & $0.0232(10)$ \\
\hline C6 & $0.40549(12)$ & $0.5928(3)$ & $0.3071(5)$ & $0.0222(10)$ \\
\hline $\mathrm{C} 7$ & $0.43060(11)$ & $0.6388(3)$ & $0.2228(5)$ & $0.0187(9)$ \\
\hline $\mathrm{C} 8$ & $0.42996(11)$ & $0.7220(3)$ & $0.2288(5)$ & $0.0211(10)$ \\
\hline C9 & $0.45401(12)$ & $0.7676(3)$ & $0.1385(5)$ & $0.0209(9)$ \\
\hline $\mathrm{C} 10$ & $0.45216(13)$ & $0.8504(3)$ & $0.1381(6)$ & $0.0297(11)$ \\
\hline $\mathrm{C} 11$ & $0.47518(14)$ & $0.8948(3)$ & $0.0521(6)$ & $0.0345(12)$ \\
\hline $\mathrm{C} 12$ & $0.50178(14)$ & $0.8572(3)$ & $0.9617(6)$ & $0.0320(12)$ \\
\hline C13 & $0.50426(12)$ & $0.7778(3)$ & $0.9587(5)$ & $0.0251(10)$ \\
\hline C14 & $0.48069(11)$ & $0.7295(3)$ & $0.0451(5)$ & $0.0208(10)$ \\
\hline $\mathrm{C} 15$ & $0.48260(11)$ & $0.6451(3)$ & $0.0437(5)$ & $0.0183(9)$ \\
\hline $\mathrm{C} 16$ & $0.45751(11)$ & $0.5999(3)$ & $0.1309(5)$ & $0.0182(9)$ \\
\hline $\mathrm{C} 17$ & $0.40520(12)$ & $0.7599(3)$ & $0.3316(5)$ & $0.0236(10)$ \\
\hline C18 & $0.38458(12)$ & $0.7882(3)$ & $0.4236(5)$ & $0.0240(10)$ \\
\hline C19 & $0.37264(13)$ & $0.7977(3)$ & $0.7765(5)$ & $0.0267(10)$ \\
\hline $\mathrm{C} 20$ & $0.39039(14)$ & $0.7159(3)$ & $0.7819(6)$ & $0.0366(12)$ \\
\hline $\mathrm{C} 21$ & $0.34373(16)$ & $0.8052(4)$ & $0.9036(6)$ & $0.0450(15)$ \\
\hline $\mathrm{C} 22$ & $0.30610(12)$ & $0.7821(3)$ & $0.5120(5)$ & $0.0276(11)$ \\
\hline $\mathrm{C} 23$ & $0.30575(14)$ & $0.6924(3)$ & $0.5249(6)$ & $0.0361(12)$ \\
\hline $\mathrm{C} 24$ & $0.29224(13)$ & $0.8091(3)$ & $0.3419(5)$ & $0.0341(12)$ \\
\hline $\mathrm{C} 25$ & $0.35153(13)$ & $0.9363(3)$ & $0.5424(5)$ & $0.0281(11)$ \\
\hline $\mathrm{C} 26$ & $0.31897(15)$ & $0.9750(3)$ & $0.6189(6)$ & $0.0375(13)$ \\
\hline $\mathrm{C} 27$ & $0.38826(15)$ & $0.9764(3)$ & $0.5943(7)$ & $0.0420(14)$ \\
\hline O1S & $0.2936(3)$ & $0.6306(6)$ & $0.9515(11)$ & $0.063(2)$ \\
\hline $\mathrm{C} 1 \mathrm{~S}$ & $0.2592(3)$ & $0.6174(10)$ & $0.049(2)$ & $0.063(2)$ \\
\hline $\mathrm{C} 2 \mathrm{~S}$ & $0.2682(4)$ & $0.5548(11)$ & $0.144(2)$ & $0.070(2)$ \\
\hline $\mathrm{C} 3 \mathrm{~S}$ & $0.3086(3)$ & $0.5405(10)$ & $0.165(2)$ & $0.064(3)$ \\
\hline $\mathrm{C} 4 \mathrm{~S}$ & $0.3245(3)$ & $0.6050(10)$ & $0.0648(19)$ & $0.056(2)$ \\
\hline
\end{tabular}


Yadav et al.

$\begin{array}{ccccc} & \mathrm{x} / \mathrm{a} & \mathrm{y} / \mathrm{b} & \mathrm{z} / \mathrm{c} & \mathrm{U}(\mathrm{eq}) \\ \mathrm{O} 1 \mathrm{~T} & 0.2739(3) & 0.6307(7) & 0.0199(14) & 0.075(2) \\ \mathrm{C} 1 \mathrm{~T} & 0.2538(3) & 0.5869(8) & 0.1513(18) & 0.056(2) \\ \mathrm{C} 2 \mathrm{~T} & 0.2721(4) & 0.5167(9) & 0.163(2) & 0.070(3) \\ \mathrm{C} 3 \mathrm{~T} & 0.3119(3) & 0.5263(9) & 0.135(2) & 0.064(3) \\ \mathrm{C} 4 \mathrm{~T} & 0.3128(3) & 0.6036(10) & 0.046(2) & 0.060(2)\end{array}$

Table S3. Bond lengths ( $\mathrm{A})$ for 5 .

\begin{tabular}{|c|c|c|c|}
\hline Si1-C18 & $1.838(4)$ & Si1-C25 & $1.880(5)$ \\
\hline Si1-C22 & $1.891(5)$ & Si1-C19 & $1.891(5)$ \\
\hline $\mathrm{C} 1-\mathrm{C} 2$ & $1.368(6)$ & C1-C15 & $1.418(6)$ \\
\hline C1-H1 & 0.95 & $\mathrm{C} 2-\mathrm{C} 2$ & $1.432(8)$ \\
\hline $\mathrm{C} 2-\mathrm{C} 3$ & $1.469(5)$ & $\mathrm{C} 3-\mathrm{C} 4$ & $1.397(6)$ \\
\hline C3-C16 & $1.424(6)$ & C4-C5 & $1.395(6)$ \\
\hline C4-H4 & 0.95 & C5-C6 & $1.364(7)$ \\
\hline C5-H5 & 0.95 & C6-C7 & $1.419(6)$ \\
\hline C6-H6 & 0.95 & C7-C8 & $1.418(6)$ \\
\hline C7-C16 & $1.437(6)$ & C8-C9 & $1.417(6)$ \\
\hline C8-C17 & $1.432(6)$ & C9-C10 & $1.411(7)$ \\
\hline C9-C14 & $1.433(6)$ & C10-C11 & $1.362(7)$ \\
\hline C10-H10 & 0.95 & C11-C12 & $1.413(7)$ \\
\hline C11-H11 & 0.95 & C12-C13 & $1.356(7)$ \\
\hline C12-H12 & 0.95 & C13-C14 & $1.414(6)$ \\
\hline C13-H13 & 0.95 & C14-C15 & $1.438(6)$ \\
\hline C15-C1 & $1.418(6)$ & C15-C16 & $1.424(6)$ \\
\hline C17-C18 & $1.205(6)$ & C19-C20 & $1.532(7)$ \\
\hline C19-C21 & $1.541(7)$ & C19-H19 & 1.0 \\
\hline $\mathrm{C} 20-\mathrm{H} 20 \mathrm{~A}$ & 0.98 & C20-H20B & 0.98 \\
\hline $\mathrm{C} 20-\mathrm{H} 20 \mathrm{C}$ & 0.98 & $\mathrm{C} 21-\mathrm{H} 21 \mathrm{~A}$ & 0.98 \\
\hline C21-H21B & 0.98 & $\mathrm{C} 21-\mathrm{H} 21 \mathrm{C}$ & 0.98 \\
\hline $\mathrm{C} 22-\mathrm{C} 23$ & $1.530(7)$ & C22-C24 & $1.543(6)$ \\
\hline C22-H22 & 1.0 & $\mathrm{C} 23-\mathrm{H} 23 \mathrm{~A}$ & 0.98 \\
\hline
\end{tabular}


Yadav et al.

\begin{tabular}{llll} 
C23-H23B & 0.98 & C23-H23C & 0.98 \\
C24-H24A & 0.98 & C24-H24B & 0.98 \\
C24-H24C & 0.98 & C25-C26 & $1.525(6)$ \\
C25-C27 & $1.530(7)$ & C25-H25 & 1.0 \\
C26-H26A & 0.98 & C26-H26B & 0.98 \\
C26-H26C & 0.98 & C27-H27A & 0.98 \\
C27-H27B & 0.98 & C27-H27C & 0.98 \\
O1S-C4S & $1.479(11)$ & O1S-C1S & $1.548(12)$ \\
C1S-C2S & $1.356(15)$ & C1S-H1S1 & 0.99 \\
C1S-H1S2 & 0.99 & C2S-C3S & $1.479(11)$ \\
C2S-H2S1 & 0.99 & C2S-H2S2 & 0.99 \\
C3S-C4S & $1.514(10)$ & C3S-H3S1 & 0.99 \\
C3S-H3S2 & 0.99 & C4S-H4S1 & 0.99 \\
C4S-H4S2 & 0.99 & O1T-C4T & $1.482(11)$ \\
O1T-C1T & $1.544(12)$ & C1T-C2T & $1.364(15)$ \\
C1T-H1T1 & 0.99 & C1T-H1T2 & 0.99 \\
C2T-C3T & $1.479(11)$ & C2T-H2T1 & 0.99 \\
C2T-H2T2 & 0.99 & C3T-C4T & $1.511(10)$ \\
C3T-H3T1 & 0.99 & 0.99 \\
C4T-H4T1 & 0.99 & 0.99 \\
\hline
\end{tabular}

Table S4. Bond angles $\left(^{\circ}\right)$ for 5.

$\begin{array}{llll}\text { C18-Si1-C25 } & 106.8(2) & \text { C18-Si1-C22 } & 106.0(2) \\ \text { C25-Si1-C22 } & 110.4(2) & \text { C18-Si1-C19 } & 107.5(2) \\ \text { C25-Si1-C19 } & 111.9(2) & \text { C22-Si1-C19 } & 113.8(2) \\ \text { C2-C1-C15 } & 124.1(4) & \text { C2-C1-H1 } & 117.9 \\ \text { C15-C1-H1 } & 117.9 & \text { C1-C2-C2 } & 119.9(5) \\ \text { C1-C2-C3 } & 122.0(4) & \text { C2-C2-C3 } & 118.2(5) \\ \text { C4-C3-C16 } & 118.9(4) & \text { C4-C3-C2 } & 121.7(4) \\ \text { C16-C3-C2 } & 119.5(4) & \text { C5-C4-C3 } & 121.4(4) \\ \text { C5-C4-H4 } & 119.3 & \text { C3-C4-H4 } & 119.3 \\ \text { C6-C5-C4 } & 120.9(4) & \text { C6-C5-H5 } & 119.5\end{array}$


Yadav et al.

\begin{tabular}{|c|c|c|c|}
\hline C4-C5-H5 & 119.5 & $\mathrm{C} 5-\mathrm{C} 6-\mathrm{C} 7$ & $120.5(4)$ \\
\hline C5-C6-H6 & 119.8 & C7-C6-H6 & 119.8 \\
\hline C8-C7-C6 & $121.4(4)$ & C8-C7-C16 & $119.5(4)$ \\
\hline C6-C7-C16 & 119.1(4) & C9-C8-C7 & $121.0(4)$ \\
\hline C9-C8-C17 & $120.0(4)$ & C7-C8-C17 & $118.9(4)$ \\
\hline C10-C9-C8 & $121.1(4)$ & C10-C9-C14 & $118.9(4)$ \\
\hline C8-C9-C14 & $119.9(4)$ & C11-C10-C9 & $121.7(4)$ \\
\hline C11-C10-H10 & 119.2 & C9-C10-H10 & 119.2 \\
\hline C10-C11-C12 & $119.3(5)$ & C10-C11-H11 & 120.3 \\
\hline C12-C11-H11 & 120.3 & C13-C12-C11 & $120.6(5)$ \\
\hline C13-C12-H12 & 119.7 & C11-C12-H12 & 119.7 \\
\hline C12-C13-C14 & $121.8(4)$ & C12-C13-H13 & 119.1 \\
\hline C14-C13-H13 & 119.1 & C13-C14-C9 & $117.6(4)$ \\
\hline C13-C14-C15 & $123.0(4)$ & C9-C14-C15 & $119.4(4)$ \\
\hline C1-C15-C16 & $117.5(4)$ & $\mathrm{C} 1-\mathrm{C} 15-\mathrm{C} 14$ & $122.3(4)$ \\
\hline C16-C15-C14 & $120.2(4)$ & C3-C16-C15 & $120.8(4)$ \\
\hline C3-C16-C7 & 119.2(4) & C15-C16-C7 & $119.9(4)$ \\
\hline C18-C17-C8 & $176.4(5)$ & C17-C18-Si1 & $177.1(4)$ \\
\hline C20-C19-C21 & $110.9(4)$ & C20-C19-Si1 & $113.2(3)$ \\
\hline C21-C19-Si1 & $112.2(3)$ & C20-C19-H19 & 106.7 \\
\hline C21-C19-H19 & 106.7 & Si1-C19-H19 & 106.7 \\
\hline C19-C20-H20A & 109.5 & C19-C20-H20B & 109.5 \\
\hline H20A-C20-H20B & 109.5 & C19-C20-H20C & 109.5 \\
\hline $\mathrm{H} 20 \mathrm{~A}-\mathrm{C} 20-\mathrm{H} 20 \mathrm{C}$ & 109.5 & $\mathrm{H} 20 \mathrm{~B}-\mathrm{C} 20-\mathrm{H} 20 \mathrm{C}$ & 109.5 \\
\hline C19-C21-H21A & 109.5 & C19-C21-H21B & 109.5 \\
\hline $\mathrm{H} 21 \mathrm{~A}-\mathrm{C} 21-\mathrm{H} 21 \mathrm{~B}$ & 109.5 & C19-C21-H21C & 109.5 \\
\hline $\mathrm{H} 21 \mathrm{~A}-\mathrm{C} 21-\mathrm{H} 21 \mathrm{C}$ & 109.5 & $\mathrm{H} 21 \mathrm{~B}-\mathrm{C} 21-\mathrm{H} 21 \mathrm{C}$ & 109.5 \\
\hline C23-C22-C24 & $111.0(4)$ & C23-C22-Si1 & $113.2(3)$ \\
\hline C24-C22-Si1 & $110.2(3)$ & $\mathrm{C} 23-\mathrm{C} 22-\mathrm{H} 22$ & 107.4 \\
\hline C24-C22-H22 & 107.4 & Si1-C22-H22 & 107.4 \\
\hline C22-C23-H23A & 109.5 & C22-C23-H23B & 109.5 \\
\hline
\end{tabular}


Yadav et al.

\begin{tabular}{|c|c|c|c|}
\hline $\mathrm{H} 23 \mathrm{~A}-\mathrm{C} 23-\mathrm{H} 23 \mathrm{~B}$ & 109.5 & C22-C23-H23C & 109.5 \\
\hline $\mathrm{H} 23 \mathrm{~A}-\mathrm{C} 23-\mathrm{H} 23 \mathrm{C}$ & 109.5 & $\mathrm{H} 23 \mathrm{~B}-\mathrm{C} 23-\mathrm{H} 23 \mathrm{C}$ & 109.5 \\
\hline C22-C24-H24A & 109.5 & C22-C24-H24B & 109.5 \\
\hline H24A-C24-H24B & 109.5 & $\mathrm{C} 22-\mathrm{C} 24-\mathrm{H} 24 \mathrm{C}$ & 109.5 \\
\hline $\mathrm{H} 24 \mathrm{~A}-\mathrm{C} 24-\mathrm{H} 24 \mathrm{C}$ & 109.5 & $\mathrm{H} 24 \mathrm{~B}-\mathrm{C} 24-\mathrm{H} 24 \mathrm{C}$ & 109.5 \\
\hline C26-C25-C27 & $111.5(4)$ & C26-C25-Si1 & $113.4(3)$ \\
\hline C27-C25-Si1 & 113.1(3) & $\mathrm{C} 26-\mathrm{C} 25-\mathrm{H} 25$ & 106.1 \\
\hline C27-C25-H25 & 106.1 & $\mathrm{Si} 1-\mathrm{C} 25-\mathrm{H} 25$ & 106.1 \\
\hline C25-C26-H26A & 109.5 & C25-C26-H26B & 109.5 \\
\hline H26A-C26-H26B & 109.5 & $\mathrm{C} 25-\mathrm{C} 26-\mathrm{H} 26 \mathrm{C}$ & 109.5 \\
\hline $\mathrm{H} 26 \mathrm{~A}-\mathrm{C} 26-\mathrm{H} 26 \mathrm{C}$ & 109.5 & H26B-C26-H26C & 109.5 \\
\hline C25-C27-H27A & 109.5 & C25-C27-H27B & 109.5 \\
\hline $\mathrm{H} 27 \mathrm{~A}-\mathrm{C} 27-\mathrm{H} 27 \mathrm{~B}$ & 109.5 & $\mathrm{C} 25-\mathrm{C} 27-\mathrm{H} 27 \mathrm{C}$ & 109.5 \\
\hline $\mathrm{H} 27 \mathrm{~A}-\mathrm{C} 27-\mathrm{H} 27 \mathrm{C}$ & 109.5 & H27B-C27-H27C & 109.5 \\
\hline C4S-O1S-C1S & $102.7(8)$ & C2S-C1S-O1S & $104.6(9)$ \\
\hline C2S-C1S-H1S1 & 110.8 & O1S-C1S-H1S1 & 110.8 \\
\hline C2S-C1S-H1S2 & 110.8 & O1S-C1S-H1S2 & 110.8 \\
\hline H1S1-C1S-H1S2 & 108.9 & C1S-C2S-C3S & $113.3(10$ \\
\hline C1S-C2S-H2S1 & 108.9 & C3S-C2S-H2S1 & 108.9 \\
\hline $\mathrm{C} 1 \mathrm{~S}-\mathrm{C} 2 \mathrm{~S}-\mathrm{H} 2 \mathrm{~S} 2$ & 108.9 & $\mathrm{C} 3 \mathrm{~S}-\mathrm{C} 2 \mathrm{~S}-\mathrm{H} 2 \mathrm{~S} 2$ & 108.9 \\
\hline $\mathrm{H} 2 \mathrm{~S} 1-\mathrm{C} 2 \mathrm{~S}-\mathrm{H} 2 \mathrm{~S} 2$ & 107.7 & C2S-C3S-C4S & $102.9(8)$ \\
\hline C2S-C3S-H3S1 & 111.2 & C4S-C3S-H3S1 & 111.2 \\
\hline C2S-C3S-H3S2 & 111.2 & C4S-C3S-H3S2 & 111.2 \\
\hline H3S1-C3S-H3S2 & 109.1 & O1S-C4S-C3S & $105.6(8)$ \\
\hline O1S-C4S-H4S1 & 110.6 & C3S-C4S-H4S1 & 110.6 \\
\hline O1S-C4S-H4S2 & 110.6 & C3S-C4S-H4S2 & 110.6 \\
\hline $\mathrm{H} 4 \mathrm{~S} 1-\mathrm{C} 4 \mathrm{~S}-\mathrm{H} 4 \mathrm{~S} 2$ & 108.7 & C4T-O1T-C1T & $103.2(8)$ \\
\hline C2T-C1T-O1T & 103.0(9) & C2T-C1T-H1T1 & 111.2 \\
\hline O1T-C1T-H1T1 & 111.2 & C2T-C1T-H1T2 & 111.2 \\
\hline O1T-C1T-H1T2 & 111.2 & H1T1-C1T-H1T2 & 109.1 \\
\hline C1T-C2T-C3T & $111.3(10)$ & C1T-C2T-H2T1 & 109.4 \\
\hline
\end{tabular}


Yadav et al.

$\begin{array}{llll}\text { C3T-C2T-H2T1 } & 109.4 & \text { C1T-C2T-H2T2 } & 109.4 \\ \text { C3T-C2T-H2T2 } & 109.4 & \text { H2T1-C2T-H2T2 } & 108.0 \\ \text { C2T-C3T-C4T } & 103.0(8) & \text { C2T-C3T-H3T1 } & 111.2 \\ \text { C4T-C3T-H3T1 } & 111.2 & \text { C2T-C3T-H3T2 } & 111.2 \\ \text { C4T-C3T-H3T2 } & 111.2 & \text { H3T1-C3T-H3T2 } & 109.1 \\ \text { O1T-C4T-C3T } & 107.0(7) & \text { O1T-C4T-H4T1 } & 110.3 \\ \text { C3T-C4T-H4T1 } & 110.3 & \text { O1T-C4T-H4T2 } & 110.3 \\ \text { C3T-C4T-H4T2 } & 110.3 & \text { H4T1-C4T-H4T2 } & 108.6\end{array}$

Table S5. Torsion angles $\left(^{\circ}\right)$ for 5.

$\begin{array}{llll}\text { C15-C1-C2-C2 } & -0.7(7) & \text { C15-C1-C2-C3 } & 179.1(4) \\ \text { C1-C2-C3-C4 } & -0.8(6) & \text { C2-C2-C3-C4 } & 179.0(4) \\ \text { C1-C2-C3-C16 } & 178.8(4) & \text { C2-C2-C3-C16 } & -1.4(7) \\ \text { C16-C3-C4-C5 } & -1.1(6) & \text { C2-C3-C4-C5 } & 178.5(4) \\ \text { C3-C4-C5-C6 } & -0.7(7) & \text { C4-C5-C6-C7 } & 1.3(7) \\ \text { C5-C6-C7-C8 } & -179.3(4) & \text { C5-C6-C7-C16 } & 0.0(6) \\ \text { C6-C7-C8-C9 } & -177.1(4) & \text { C16-C7-C8-C9 } & 3.6(6) \\ \text { C6-C7-C8-C17 } & 4.6(6) & \text { C16-C7-C8-C17 } & -174.7(4) \\ \text { C7-C8-C9-C10 } & 176.8(4) & \text { C17-C8-C9-C10 } & -4.9(7) \\ \text { C7-C8-C9-C14 } & -3.0(6) & \text { C17-C8-C9-C14 } & 175.3(4) \\ \text { C8-C9-C10-C11 } & 179.8(5) & \text { C14-C9-C10-C11 } & -0.4(7) \\ \text { C9-C10-C11-C12 } & -0.4(8) & \text { C10-C11-C12-C13 } & 0.7(8) \\ \text { C11-C12-C13-C14 } & -0.1(8) & \text { C12-C13-C14-C9 } & -0.6(7) \\ \text { C12-C13-C14-C15 } & 179.8(5) & \text { C10-C9-C14-C13 } & 0.9(6) \\ \text { C8-C9-C14-C13 } & -179.3(4) & \text { C10-C9-C14-C15 } & -179.5(4) \\ \text { C8-C9-C14-C15 } & 0.3(6) & \text { C13-C14-C15-C1 } & 2.2(7) \\ \text { C9-C14-C15-C1 } & -177.4(4) & \text { C13-C14-C15-C16 } & -178.7(4) \\ \text { C9-C14-C15-C16 } & 1.7(6) & \text { C4-C3-C16-C15 } & -178.6(4) \\ \text { C2-C3-C16-C15 } & 1.8(6) & \text { C4-C3-C16-C7 } & 2.3(6) \\ \text { C2-C3-C16-C7 } & -177.3(4) & \text { C1-C15-C16-C3 } & -1.0(6) \\ \text { C14-C15-C16-C3 } & 179.9(4) & \text { C1-C15-C16-C7 } & 178.1(4)\end{array}$


Yadav et al.

\begin{tabular}{llll} 
C14-C15-C16-C7 & $-1.0(6)$ & C8-C7-C16-C3 & $177.5(4)$ \\
C6-C7-C16-C3 & $-1.8(6)$ & C8-C7-C16-C15 & $-1.6(6)$ \\
C6-C7-C16-C15 & $179.1(4)$ & C18-Si1-C19-C20 & $-37.2(4)$ \\
C25-Si1-C19-C20 & $-154.1(3)$ & C22-Si1-C19-C20 & $79.8(4)$ \\
C18-Si1-C19-C21 & $-163.6(4)$ & C25-Si1-C19-C21 & $79.4(4)$ \\
C22-Si1-C19-C21 & $-46.6(4)$ & C18-Si1-C22-C23 & $62.5(4)$ \\
C25-Si1-C22-C23 & $177.8(3)$ & C19-Si1-C22-C23 & $-55.4(4)$ \\
C18-Si1-C22-C24 & $-62.4(4)$ & C25-Si1-C22-C24 & $52.9(4)$ \\
C19-Si1-C22-C24 & $179.7(3)$ & C18-Si1-C25-C26 & $163.8(3)$ \\
C22-Si1-C25-C26 & $49.1(4)$ & C19-Si1-C25-C26 & $-78.8(4)$ \\
C18-Si1-C25-C27 & $-67.9(4)$ & C22-Si1-C25-C27 & $177.3(3)$ \\
C19-Si1-C25-C27 & $49.5(4)$ & C4S-O1S-C1S-C2S & $-31.6(16)$ \\
O1S-C1S-C2S-C3S & $20 .(2)$ & C1S-C2S-C3S-C4S & $0 .(2)$ \\
C1S-O1S-C4S-C3S & $31.5(14)$ & C2S-C3S-C4S-O1S & $-20.5(17)$ \\
C4T-O1T-C1T-C2T & $-34.4(14)$ & O1T-C1T-C2T-C3T & $34.4(16)$ \\
C1T-C2T-C3T-C4T & $-20.4(19)$ & C1T-O1T-C4T-C3T & $22.3(17)$ \\
C2T-C3T-C4T-O1T & $-3.3(19)$ & & \\
\hline
\end{tabular}

Table S6. Anisotropic atomic displacement parameters $\left(\AA^{2}\right)$ for 5. The anisotropic atomic displacement factor exponent takes the form: $-2 \pi^{2}\left[h^{2} a^{* 2} U^{11}+\ldots+2 h k a^{*} b^{*} U^{12}\right]$

$\begin{array}{lllllll} & \mathrm{U}_{11} & \mathrm{U}_{22} & \mathrm{U}_{33} & \mathrm{U}_{23} & \mathrm{U}_{13} & \mathrm{U}_{12} \\ \text { Si1 } & 0.0189(6) & 0.0244(6) & 0.0203(6) & -0.0013(5) & 0.0049(4) & 0.0037(5) \\ \text { C1 } & 0.016(2) & 0.026(2) & 0.015(2) & -0.0021(17) & 0.0025(16) & -0.0035(18) \\ \text { C2 } & 0.014(2) & 0.028(2) & 0.012(2) & -0.0031(17) & -0.0011(15) & 0.0030(18) \\ \text { C3 } & 0.015(2) & 0.023(2) & 0.012(2) & -0.0046(17) & -0.0007(15) & 0.0037(17) \\ \text { C4 } & 0.019(2) & 0.027(3) & 0.021(2) & -0.0028(19) & 0.0049(17) & -0.0008(19) \\ \text { C5 } & 0.015(2) & 0.036(3) & 0.019(2) & 0.0002(19) & 0.0047(17) & -0.0005(19) \\ \text { C6 } & 0.016(2) & 0.034(3) & 0.017(2) & -0.0031(19) & 0.0020(16) & 0.0030(19) \\ \text { C7 } & 0.014(2) & 0.027(2) & 0.015(2) & -0.0020(18) & 0.0007(16) & 0.0005(18) \\ \text { C8 } & 0.016(2) & 0.029(3) & 0.018(2) & -0.0002(19) & 0.0022(16) & 0.0048(19) \\ \text { C9 } & 0.016(2) & 0.024(2) & 0.024(2) & -0.0006(19) & 0.0041(17) & 0.0016(18) \\ \text { C10 } & 0.026(2) & 0.029(3) & 0.034(3) & -0.007(2) & 0.006(2) & 0.002(2)\end{array}$




\begin{tabular}{|c|c|c|c|c|c|c|}
\hline & $\mathrm{U}_{11}$ & $\mathrm{U}_{22}$ & $\mathrm{U}_{33}$ & $\mathrm{U}_{23}$ & $\mathrm{U}_{13}$ & $\mathrm{U}_{12}$ \\
\hline $\mathrm{C} 11$ & $0.034(3)$ & $0.023(3)$ & $0.048(3)$ & $-0.003(2)$ & $0.015(2)$ & $0.000(2)$ \\
\hline $\mathrm{C} 12$ & $0.031(3)$ & $0.024(3)$ & $0.042(3)$ & $-0.002(2)$ & $0.015(2)$ & $0.000(2)$ \\
\hline C13 & $0.021(2)$ & $0.028(3)$ & $0.027(2)$ & $-0.003(2)$ & $0.0093(18)$ & $0.000(2)$ \\
\hline $\mathrm{C} 14$ & $0.016(2)$ & $0.027(3)$ & $0.019(2)$ & $-0.0051(18)$ & $0.0014(17)$ & $0.0039(18)$ \\
\hline $\mathrm{C} 15$ & $0.014(2)$ & $0.026(2)$ & $0.014(2)$ & $-0.0031(17)$ & $0.0004(16)$ & $0.0043(17)$ \\
\hline $\mathrm{C} 16$ & $0.014(2)$ & $0.026(2)$ & $0.014(2)$ & $-0.0025(17)$ & $-0.0003(15)$ & $0.0030(18)$ \\
\hline $\mathrm{C} 17$ & $0.020(2)$ & $0.028(3)$ & $0.023(2)$ & $-0.0001(19)$ & $0.0009(18)$ & $0.0008(19)$ \\
\hline $\mathrm{C} 18$ & $0.024(2)$ & $0.023(2)$ & $0.025(2)$ & $-0.0044(19)$ & $0.0042(19)$ & $0.0000(19)$ \\
\hline C19 & $0.031(3)$ & $0.026(3)$ & $0.023(2)$ & $-0.0032(19)$ & $-0.0030(19)$ & $0.000(2)$ \\
\hline $\mathrm{C} 20$ & $0.035(3)$ & $0.039(3)$ & $0.035(3)$ & $0.008(2)$ & $0.001(2)$ & $0.009(2)$ \\
\hline $\mathrm{C} 21$ & $0.060(4)$ & $0.052(4)$ & $0.024(3)$ & $0.004(2)$ & $0.014(2)$ & $0.015(3)$ \\
\hline $\mathrm{C} 22$ & $0.022(2)$ & $0.036(3)$ & $0.026(2)$ & $0.001(2)$ & $0.0072(18)$ & $0.007(2)$ \\
\hline $\mathrm{C} 23$ & $0.026(3)$ & $0.040(3)$ & $0.043(3)$ & $0.000(2)$ & $0.004(2)$ & $-0.001(2)$ \\
\hline $\mathrm{C} 24$ & $0.030(3)$ & $0.045(3)$ & $0.027(2)$ & $-0.002(2)$ & $-0.002(2)$ & $-0.002(2)$ \\
\hline $\mathrm{C} 25$ & $0.029(3)$ & $0.030(3)$ & $0.025(2)$ & $0.001(2)$ & $0.0050(19)$ & $0.004(2)$ \\
\hline $\mathrm{C} 26$ & $0.046(3)$ & $0.031(3)$ & $0.036(3)$ & $-0.004(2)$ & $0.008(2)$ & $0.009(2)$ \\
\hline $\mathrm{C} 27$ & $0.047(3)$ & $0.027(3)$ & $0.053(3)$ & $0.000(3)$ & $0.007(3)$ & $-0.005(2)$ \\
\hline O1S & $0.045(4)$ & $0.077(5)$ & $0.069(5)$ & $0.024(4)$ & $0.006(4)$ & $-0.022(4)$ \\
\hline $\mathrm{C} 1 \mathrm{~S}$ & $0.032(5)$ & $0.075(5)$ & $0.081(5)$ & $0.014(4)$ & $0.005(4)$ & $-0.008(4)$ \\
\hline $\mathrm{C} 2 \mathrm{~S}$ & $0.044(5)$ & $0.080(5)$ & $0.086(5)$ & $0.020(5)$ & $0.003(4)$ & $-0.011(5)$ \\
\hline $\mathrm{C} 3 \mathrm{~S}$ & $0.043(4)$ & $0.072(5)$ & $0.076(5)$ & $0.014(5)$ & $0.009(4)$ & $-0.001(4)$ \\
\hline $\mathrm{C} 4 \mathrm{~S}$ & $0.037(5)$ & $0.065(5)$ & $0.065(5)$ & $0.017(4)$ & $0.006(4)$ & $0.002(4)$ \\
\hline $\mathrm{O} 1 \mathrm{~T}$ & $0.056(5)$ & $0.085(4)$ & $0.084(4)$ & $0.013(4)$ & $-0.001(4)$ & $0.006(4)$ \\
\hline $\mathrm{C} 1 \mathrm{~T}$ & $0.029(5)$ & $0.067(6)$ & $0.073(5)$ & $0.017(5)$ & $0.007(4)$ & $0.006(4)$ \\
\hline $\mathrm{C} 2 \mathrm{~T}$ & $0.043(4)$ & $0.073(5)$ & $0.093(5)$ & $0.022(5)$ & $0.000(4)$ & $-0.001(5)$ \\
\hline $\mathrm{C} 3 \mathrm{~T}$ & $0.045(4)$ & $0.069(5)$ & $0.079(5)$ & $0.015(5)$ & $0.012(4)$ & $0.007(4)$ \\
\hline $\mathrm{C} 4 \mathrm{~T}$ & $0.041(5)$ & $0.069(5)$ & $0.070(5)$ & $0.017(4)$ & $0.007(4)$ & $0.011(4)$ \\
\hline
\end{tabular}

Table S7. Hydrogen atomic coordinates and isotropic atomic displacement parameters $\left(\AA^{2}\right)$ for 5 . 


\begin{tabular}{|c|c|c|c|c|}
\hline & $\mathrm{x} / \mathrm{a}$ & $y / b$ & $\mathrm{z} / \mathrm{c}$ & $\mathrm{U}(\mathrm{eq})$ \\
\hline H1 & 0.4740 & 0.3665 & 0.0997 & 0.023 \\
\hline $\mathrm{H} 4$ & 0.4339 & 0.4186 & 0.2135 & 0.026 \\
\hline H5 & 0.3895 & 0.4827 & 0.3563 & 0.028 \\
\hline H6 & 0.3876 & 0.6179 & 0.3682 & 0.027 \\
\hline H10 & 0.4344 & 0.8759 & 0.1993 & 0.036 \\
\hline H11 & 0.4734 & 0.9505 & 0.0530 & 0.041 \\
\hline H12 & 0.5181 & 0.8879 & -0.0976 & 0.038 \\
\hline H13 & 0.5223 & 0.7538 & -0.1032 & 0.03 \\
\hline H19 & 0.3929 & 0.8360 & 0.8070 & 0.032 \\
\hline $\mathrm{H} 20 \mathrm{~A}$ & 0.4005 & 0.7048 & 0.8914 & 0.055 \\
\hline $\mathrm{H} 20 \mathrm{~B}$ & 0.4104 & 0.7142 & 0.7081 & 0.055 \\
\hline $\mathrm{H} 20 \mathrm{C}$ & 0.3716 & 0.6765 & 0.7497 & 0.055 \\
\hline $\mathrm{H} 21 \mathrm{~A}$ & 0.3235 & 0.7679 & 0.8793 & 0.068 \\
\hline $\mathrm{H} 21 \mathrm{~B}$ & 0.3338 & 0.8588 & 0.9022 & 0.068 \\
\hline $\mathrm{H} 21 \mathrm{C}$ & 0.3555 & 0.7938 & 1.0102 & 0.068 \\
\hline $\mathrm{H} 22$ & 0.2883 & 0.8029 & 0.5887 & 0.033 \\
\hline $\mathrm{H} 23 \mathrm{~A}$ & 0.2814 & 0.6724 & 0.4850 & 0.054 \\
\hline $\mathrm{H} 23 \mathrm{~B}$ & 0.3106 & 0.6769 & 0.6375 & 0.054 \\
\hline $\mathrm{H} 23 \mathrm{C}$ & 0.3250 & 0.6703 & 0.4605 & 0.054 \\
\hline $\mathrm{H} 24 \mathrm{~A}$ & 0.3104 & 0.7941 & 0.2650 & 0.051 \\
\hline $\mathrm{H} 24 \mathrm{~B}$ & 0.2892 & 0.8663 & 0.3410 & 0.051 \\
\hline $\mathrm{H} 24 \mathrm{C}$ & 0.2684 & 0.7840 & 0.3117 & 0.051 \\
\hline $\mathrm{H} 25$ & 0.3475 & 0.9456 & 0.4242 & 0.034 \\
\hline $\mathrm{H} 26 \mathrm{~A}$ & 0.3222 & 0.9699 & 0.7360 & 0.056 \\
\hline H26B & 0.2958 & 0.9493 & 0.5802 & 0.056 \\
\hline $\mathrm{H} 26 \mathrm{C}$ & 0.3179 & 1.0308 & 0.5898 & 0.056 \\
\hline $\mathrm{H} 27 \mathrm{~A}$ & 0.3876 & 1.0311 & 0.5574 & 0.063 \\
\hline H27B & 0.4087 & 0.9487 & 0.5470 & 0.063 \\
\hline $\mathrm{H} 27 \mathrm{C}$ & 0.3920 & 0.9752 & 0.7117 & 0.063 \\
\hline H1S1 & 0.2541 & 0.6643 & 0.1144 & 0.075 \\
\hline
\end{tabular}




$\begin{array}{lcccc} & \mathrm{x} / \mathrm{a} & \mathrm{y} / \mathrm{b} & \mathrm{z} / \mathrm{c} & \mathrm{U}(\mathrm{eq}) \\ \mathrm{H} 1 \mathrm{~S} 2 & 0.2370 & 0.6058 & -0.0232 & 0.075 \\ \text { H2S1 } & 0.2559 & 0.5073 & 0.0975 & 0.084 \\ \text { H2S2 } & 0.2585 & 0.5636 & 0.2513 & 0.084 \\ \text { H3S1 } & 0.3150 & 0.4879 & 0.1241 & 0.076 \\ \text { H3S2 } & 0.3177 & 0.5449 & 0.2790 & 0.076 \\ \text { H4S1 } & 0.3334 & 0.6493 & 0.1339 & 0.067 \\ \text { H4S2 } & 0.3454 & 0.5849 & 0.0058 & 0.067 \\ \text { H1T1 } & 0.2561 & 0.6158 & 0.2545 & 0.067 \\ \text { H1T2 } & 0.2272 & 0.5793 & 0.1188 & 0.067 \\ \text { H2T1 } & 0.2607 & 0.4797 & 0.0822 & 0.084 \\ \text { H2T2 } & 0.2695 & 0.4940 & 0.2706 & 0.084 \\ \text { H3T1 } & 0.3209 & 0.4828 & 0.0696 & 0.077 \\ \text { H3T2 } & 0.3271 & 0.5287 & 0.2383 & 0.077 \\ \text { H4T1 } & 0.3276 & 0.6427 & 0.1107 & 0.072 \\ \text { H4T2 } & 0.3240 & 0.5965 & -0.0577 & 0.072\end{array}$

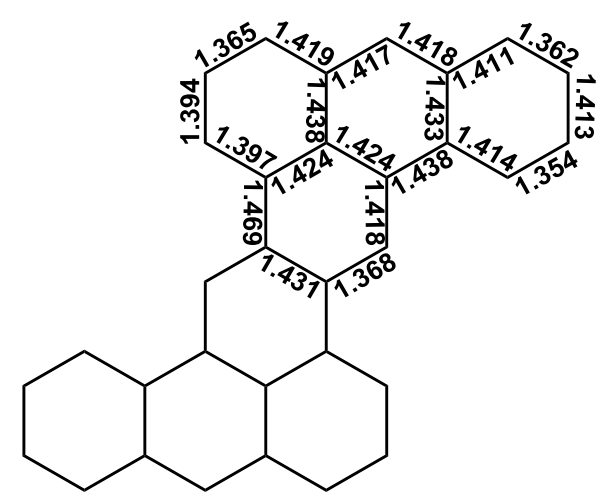

Figure S11. DBZ backbone of 5 with bond lengths representation.

\section{DFT Calculations}

Density functional theory calculations (CAM-B3LYP/6-31G(d,p), using the crystal structure, were performed in the gas phase with Gaussian 09 package utilizing a high performance computing cluster facility of NUS. ${ }^{2}$ Diradical character index $\left(y_{0}\right)$ was determined on the basis of the LUMO occupation number $(n)\left(n_{\mathrm{LUMO}}=0.67657\right.$ for $\left.\mathbf{5}\right)$ in natural orbital analysis, for the UCAM-B3LYP/6-31G(d.p) optimized singlet geometry. The $y_{0}$ is formally expressed as $y_{0}=1-\left(2 T /\left(1+T^{2}\right)\right)$, where $T$ is represented by using the occupation numbers of UCAM- 
Yadav et al.

B3LYP natural orbitals as: $T=\left(n_{\mathrm{HOMO}}-n_{\mathrm{LUMO}}\right) / 2$. A molecule with $y_{0}=0$ indicates a closedshell structure, whereas a molecule with $y_{0}=1$ implies a pure diradical structure. Any intermediate value of $y_{0}$ refers to diradicaloid (i.e. diradical like) structures.

Table S8. Relative energies of optimized geometries.

\begin{tabular}{|c|c|c|c|}
\hline Compound & Theory level/Basis set & Hartree $^{a}$ & Kcal/mol \\
\hline \multirow[t]{3}{*}{5} & Singlet CAM-B3LYP/6-31G(d,p) & -2670.502872 & -1675740.55 \\
\hline & Singlet UCAM-B3LYP/6-31G(d,p) & -2670.513273 & -1675747.07 \\
\hline & Triplet UCAM-B3LYP/6-31G(d,p) & -2670.506153 & -1675742.61 \\
\hline
\end{tabular}

${ }^{a} 1 \overline{\text { Hartree }=627.5 \mathrm{kcal} / \mathrm{mol}}$

Table S9. Theoretical NHOMO energy levels of 5 at UCAM-B3LYP/6-31G(d,p).

\begin{tabular}{||c||c||c||c||}
\hline Compound & SOMO $\alpha$ & NHOMO $\alpha-1$ & NHOMO $\alpha-2$ \\
\hline $\mathbf{5}$ & $-5.69 \mathrm{eV}$ & $-7.00 \mathrm{eV}$ & $-7.63 \mathrm{eV}$ \\
\hline
\end{tabular}

\section{References:}

(1) Bain, G. A.; Berry, J. F. J. Chem. Educ. 2008, 85, 532.

(2) Gaussian 09, Revision D.01, Frisch, M. J.; Trucks, G. W.; Schlegel, H. B.; Scuseria, G.

E.; Robb, M. A.; Cheeseman, J. R.; Scalmani, G.; Barone, V.; Mennucci, B.; Petersson, G. A.; Nakatsuji, H.; Caricato, M.; Li, X.; Hratchian, H. P.; Izmaylov, A. F.; Bloino, J.; Zheng, G.; Sonnenberg, J. L.; Hada, M.; Ehara, M.; Toyota, K.; Fukuda, R.; Hasegawa, J.; Ishida, M.; Nakajima, T.; Honda, Y.; Kitao, O.; Nakai, H.; Vreven, T.; Montgomery, J. A., Jr.; Peralta, J. E.; Ogliaro, F.; Bearpark, M.; Heyd, J. J.; Brothers, E.; Kudin, K. N.; Staroverov, V. N.; Kobayashi, R.; Normand, J.; Raghavachari, K.; Rendell, A.; Burant, J. C.; Iyengar, S. S.; Tomasi, J.; Cossi, M.; Rega, N.; Millam, J. M.; Klene, M.; Knox, J. E.; Cross, J. B.; Bakken, V.; Adamo, C.; Jaramillo, J.; Gomperts, R.; Stratmann, R. E.; Yazyev, O.; Austin, A. J.; Cammi, R.; Pomelli, C.; Ochterski, J. W.; Martin, R. L.; Morokuma, K.; Zakrzewski, V. G.; Voth, G. A.; Salvador, P.; Dannenberg, J. J.; Dapprich, S.; Daniels, A. D.; Farkas, Ö.; Foresman, J. B.; Ortiz, J. V.; Cioslowski, J.; Fox, D. J. Gaussian, Inc., Wallingford CT, 2013. 